\title{
Safety and Cost-Efficacy of Surgical Fixation of Isolated Ankle Fractures in a Free-Standing Ambulatory Surgical Center (ASC) vs. Hospital-Owned Outpatient Facility (HOPD) \\ Azeem Tariq Malik, MBBS, Safdar N Khan, MD, Carmen E Quatman, MD, PhD, Thuan Ly, MD, Laura Phieffer, MD
}

\section{Introduction}

Free-standing Ambulatory Surgical Centers (ASCs) typically provide uncomplicated surgical procedures in a non-hospital setting, and function as 'focused factories'. Despite an increasing interest towards outpatient surgery, few studies have compared the safety and cost-savings associated with surgical fixation of isolated ankle fractures in a freestanding ASC vs. a Hospital-owned Outpatient Facility (HOPD).

\section{Objectives}

To understand differences in 90day Complications and Costs in patients undergoing ORIF for ankle fractures in a free-standing ASC vs. a HOPD.

\section{Methods}

- 2007-2014 Humana Claims Database

Patients undergoing ORIF for isolated uni-malleolar, bimalleolar and tri-malleolar ankle fractures in an outpatient setting. Location of Surgery: FreeStanding ASC or HOPD

Propensity-score matching and multi-variate regression analyses were used to compare outcomes.

\section{Results}

A total of $4,832(80.1 \%)$ ankle fractures treated in a HOPD and 1,198 (19.9\%) in a free-standing ASC.

Following matching, a total of 1,138 patients in each cohort.

Table 1: Baseline demographic and clinical characteristics of the matched study groups.

\begin{tabular}{|l|c|c|c|}
\hline & $\begin{array}{c}\text { HOPD } \\
(\mathrm{N}=1,138)\end{array}$ & $\begin{array}{c}\text { ASC } \\
(\mathrm{N}=1,138)\end{array}$ & P-value \\
\hline Age & $481(42.3 \%)$ & $460(40.4 \%)$ & 0.781 \\
$<50$ & $405(25.9 \%)$ & $309(27.2 \%)$ & \\
$50-64$ & $295)$ & \\
$65-79$ & $328(28.8 \%)$ & $338(29.7 \%)$ & \\
$\geq 80$ & $34(8.9 \%)$ & $31(8.9 \%)$ & \\
\hline Gender & & & 0.419 \\
Female & $678(59.6 \%)$ & $659(57.9 \%)$ & \\
Male & $460(40.4 \%)$ & $479(42.1 \%)$ & \\
\hline Race & & & 0.193 \\
White & $475(41.7 \%)$ & $464(40.8 \%)$ & \\
Black & $25(2.2 \%)$ & $34(3.0 \%)$ & \\
Other & $6(0.5 \%)$ & $14(1.2 \%)$ & \\
Unknown & $632(55.5 \%)$ & $626(55.0 \%)$ & \\
\hline Region & & & 0.204 \\
Midwest & $235(20.7 \%)$ & $221(19.4 \%)$ & \\
Northeast & $8(0.7 \%)$ & $11(0.9 \%)$ & \\
South & $780(68.5 \%)$ & $761(66.9 \%)$ & \\
West & $115(10.1 \%)$ & $145(12.7 \%)$ & \\
\hline Fracture & & & 0.923 \\
Uni-Mall & $645(56.7 \%)$ & $636(55.9 \%)$ & \\
Bi-Mall & $334(29.3 \%)$ & $342(30.1 \%)$ & \\
Tri-Mall & $159(14.0 \%)$ & $160(14.1 \%)$ & \\
\hline Mean ECI & $4.2 \pm 4.2$ & $4.4 \pm 4.4$ & 0.268 \\
\hline
\end{tabular}

No significant differences between ASCs vs. HOPDs in 90-day Outcomes

\begin{tabular}{|l|c|c|c|c|}
\hline Outcome & HOPD & ASC & $\begin{array}{c}\text { Adjusted OR } \\
{[95 \% \text { CI] }}\end{array}$ & P-value \\
\hline $\begin{array}{l}\text { Any 90-day } \\
\text { Complication }\end{array}$ & $\begin{array}{c}110 \\
(9.7 \%)\end{array}$ & $87(7.6 \%)$ & $0.73[0.54-1.00]$ & 0.050 \\
\hline SSI & $34(2.8 \%)$ & $27(2.4 \%)$ & $0.72[0.42-1.20]$ & 0.210 \\
\hline Pneumonia & $8(0.7 \%)$ & $9(0.8 \%)$ & $0.92[0.32-2.68]$ & 0.883 \\
\hline $\begin{array}{l}\text { Thrombo- } \\
\text { embolic } \\
\text { complications }\end{array}$ & $16(1.4 \%)$ & $15(1.3 \%)$ & $0.90[0.43-1.85]$ & 0.769 \\
\hline Sepsis & $6(0.5 \%)$ & $7(0.6 \%)$ & $0.81[0.22-3.05]$ & 0.753 \\
\hline Acute MI & $3(0.3 \%)$ & $2(0.2 \%)$ & $0.20[0.07-2.04]$ & 0.226 \\
\hline $\begin{array}{l}\text { Acute renal } \\
\text { failure }\end{array}$ & $14(1.2 \%)$ & $11(1.0 \%)$ & $0.49[0.19-1.21]$ & 0.132 \\
\hline UTI & $45(3.9 \%)$ & $40(3.5 \%)$ & $0.56[0.55-1.38]$ & 0.560 \\
\hline $\begin{array}{l}\text { Pressure } \\
\text { Ulcer }\end{array}$ & $5(0.4 \%)$ & $3(0.3 \%)$ & $0.43[0.06-2.23]$ & 0.345 \\
\hline $\begin{array}{l}\text { Clostridium } \\
\text { Difficile }\end{array}$ & $2(0.2 \%)$ & $2(0.2 \%)$ & $0.56[0.02-9.21]$ & 0.690 \\
\hline $\begin{array}{l}\text { 90-day ED } \\
\text { visits }\end{array}$ & 109 & 100 & $0.86[0.64-1.16]$ & 0.331 \\
\hline $\begin{array}{l}\text { 90-day } \\
\text { Readmissions }\end{array}$ & $59(5.2 \%)$ & $53(4.7 \%)$ & $0.79[0.53-1.18]$ & 0.251 \\
\hline
\end{tabular}

\section{Conclusion}

Using national administrative claims of Commercial insurance beneficiaries, the results of the study show that performing surgical fixation of ankle fractures in a free-standing ASC is a safe and costeffective option in a carefully selected patient population. 